\title{
Valentin A. Fabrikant: negative absorption, his 1951 patent application for amplification of electromagnetic radiation (ultraviolet, visible, infrared and radio spectral regions) and his experiments
}

Svetlana G. Lukishova

sluk@lle.rochester.edu
The Institute of Optics, University of Rochester, Wilmot building, 275 Hutchison Rd., Rochester NY 14627, USA

This paper is devoted to Moscow physicist Valentin A. Fabrikant who is known for his 1939 thesis with suggestion of experiments on light amplification directly proving the existence of negative absorption in gas discharge, his 1951 patent application (jointly with M. M. Vudynsky and F. A. Butaeva) for amplification of electromagnetic radiation (ultraviolet, visible, infrared and radio spectral regions), and his experimental attempts (jointly with F. A. Butaeva) to observe light amplification in gas discharge (paper submission of December 1957). [DOI: $10.2971 /$ jeos.2010.10045s]

Keywords: Fabrikant, negative absorption, light amplification

\section{INTRODUCTION}

Soviet, in particular Moscow, physicists, are well-known for their contributions to the birth of quantum electronics. In 1964 Nikolaj G. Basov and Alexander M. Prokhorov together with Charles H. Townes were awarded the 1964 Nobel Prize "for fundamental work in the field of quantum electronics, which has led to the construction of oscillators and amplifiers based on the maser-laser principle". Their contributions before and after the Nobel Prize, showing the blossoming of quantum electronics in Russia, were discussed in [1]. The history of quantum electronics research in the Soviet Union was outlined in [2].

Among Russian contributors to quantum electronics in its early years, Valentin A. Fabrikant is less known although the authors of main books on the birth of quantum electronics, e.g., [3]-[6] mention his name and his main results. The present paper was written at the request of A. E. Siegman using the materials provided by Fabrikant's relatives and E. I. Pogrebysskaya (S. I. Vavilov Institute for the History of Science and Technology), the author of publication [7] about Fabrikant. The author of present paper (S. L.) carried out her Ph.D. on laser physics at the Moscow P. N. Lebedev Physical Institute under co-supervision of A. M. Prokhorov and worked more than 20 years in the Russian Academy of Sciences before moving to the U.S. in 1997.

This paper is organized as follows. Section 2 describes Fabrikant's 1939 Doctoral thesis and its section about negative absorption. Section 3 is devoted to the history of negative absorption. Fabrikant's et al. 1951 patent application is discussed in Section 4. Section 5 describes Fabrikant and Butaeva's experimental attempts to obtain light amplification in a gas discharge. Section 6 is a short biography of Fabrikant. His main contributions to prelaser quantum electronics research are listed in the Conclusion (Section 7).
2 FABRIKANT'S 1939 DOCTORAL THESIS: SECTION "ON EXPERIMENTAL EVIDENCE FOR THE EXISTENCE OF NEGATIVE ABSORPTION"

"On experimental evidence for the existence of negative absorption" is the title of one section of Valentin A. Fabrikant's Doctoral thesis entitled "The emission mechanism of a gas discharge" which he defended in 1939 at the P. N. Lebedev Physical Institute although he was affiliated with two other institutes outside the Soviet Academy of Sciences (at this time "negative absorption" terminology was used more often than "stimulated (induced) emission"). This doctoral thesis is the second level of Soviet Degree (in addition to the Ph.D. level, Candidate of Science Degree) which corresponds to a full Professor level in the USA. After his defense, Fabrikant received the full Professor title. Early in 1935, Academician S. I. Vavilov [8]-[10] in his reference letter about Fabrikant's scientific work wrote [7] "....The level of a Candidate, in my opinion, has been passed by V. A. Fabrikant a long time ago, and should an appropriate government order be issued, he may, definitely, receive his Candidate of Physics Degree without thesis defense. The scientific weight of V. A. Fabrikant, his outstanding and wide erudition allow me already right now to consider him deserving a Professor's title". This led V. A. Fabrikant to receive the Ph.D. degree (Candidate of Science) without writing and defending a Ph.D. thesis in the same year (1935). According to the documentation available from Fabrikant's Doctoral thesis defense in 1939, Vavilov, who was Chair of the Defense Committee, summarized the result of this defense [7]: "Today thesis defense appears to be in our physical world the event, we can say, outstanding". One of three scientists who had to read his thesis carefully and submit the required letter about it ("opponents") said during the discussion [7] "You with your group are, in essence, the strongest group in the Soviet Union (in this particular field - S. L.), and people wait for your works". He was speaking of the whole thesis 


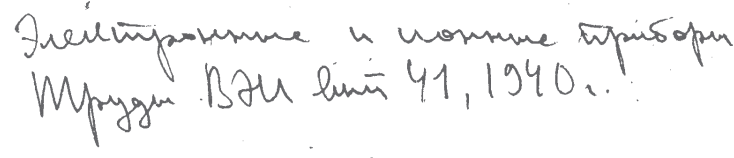

B. А. ФАБРИKАHT

МЕХАНИЗМ ИЗЛУЧЕНИЯ ГАЗОВОГО РАЗРЯДА

\section{I. ВВЕДЕНИЕ}

Настоящая статья представляет собой сжатую сводку результатов, полученных в ряде работ по изучению разряда.

При этом мы не будем останавливаться на подробностях экспериментальной методики и сосредоточим все внимание на существе полученных результатов и на их взаимной связи.

Экопериментальная методика подробно описана в уже опубли кованных нами работах. На эти работы при дальнейшем изложении будут делаться ссылки.

Изучение оптических свойств газового разряда представляе' значительный интерес по четырем основным. причинам:

1) газовый разряд является одним из наиболее экономичных и мощных источников излучения во всех вбластях спектра;

2) оптические процессы играют сущестенную роль в меха-

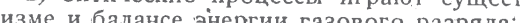

3) по оптический свойствам газового разряда можно судить об его электрокинетических характеристиках;

4) в ряде случаев изучение оптических свойств разряда по3воляет определить элементарные атомные константы.

Первые две причины придают самостоятельный интерес оптическим характеристикам разряда, последние две причины делаю изучение оптических свойств разряда важным методом для

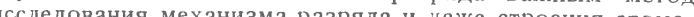

мы до сих пор ограничивались исследован

Мы до сих пор ограничивались исследованием лишь положительного столба разряда (в чистых парах и газах), оптические свойства которого нашли наиболее широкое практическое при менение.

В дальнейшем предполагается детальное исследование катодных и анодных частей разряда. Здесь особый интерес предста вит применение оптических методов к исследованию механизи разряда.

Положительный столб является наиболее простым объектом для оптических исследований, но и для него еще далеко не все вопросы, связанные с механизмом излучения, выяснены с долж

2.36

FIG. 1 Page 1 of Fabrikant"s 1940 publication of his 1939 Doctor of Science thesis.

which contained data on spectral properties and intensities of gas discharges in a wide interval of currents and pressures, analysis of elementary processes connected with exited atoms and first quantative data on second kind collisions between excited atoms and electrons, as well as connection between elementary processes and macroscopic optical characteristics. Fabrikant had started the work on gas discharge optical properties in 1932.

The same text of his 1939 thesis, was published in 1940 in the Proceedings (Trudy) of All-Union Electro-Technical Institute (VEI) with which Fabrikant was affiliated [11]. This text comprises the results of Fabrikant's 24 papers published in leading Soviet journals. Figure 1 is the first page of this paper with the title and with Fabrikant's hand-writing of the journal title, year and volume of Trudy VEI. The titles of five chapters of this paper are as follows: Chapter I. Introduction; Chapter II. Excitation of spectral lines under first-kind collisions between electrons and normal atoms: a) Equation for radiation intensity; b) Radiation of sodium lamp; c) Nonelastic losses in low pressure mercury discharge; d) Some general conclusion from the equation of number of excitation events; e) Influence of longitudinal magnetic field on gas discharge; Chapter III. Influence of second-kind processes on a positive column of gas discharge: a) Theory of excitation of metastable atoms in a positive column of gas discharge; b) Experimental measurements of concentration of metastable atoms; c) Discussion of the experimental data (including both own results and literature review - S. L.); d) Excitation of radiating atoms in a positive column of gas discharge; e) Radiation quenching in discharge; f) On experimental evidence for the existence of negative absorption (a bold font is specially used by S. L.); c) Step excitation; Chapter IV. Positive column of discharge as temperature (thermal equilibrium $-\mathrm{S}$. L.) radiation source: a) Boltzmann radiation source; b) High-pressure mercury discharge as a black-body radiation source; c) On spectra of very high-pressure mercury discharge; Chapter V. Main results: 1. Discharge radiation at low densities of gas and electrons; 2. Influence of second-kind processes on discharge radiation; 3. Gas discharge as thermal equilibrium source.

The section on "negative absorption" from this published in 1940 60-journal-page thesis [11] is later translated into English and reprinted in the book [12], containing both English translations and Russian originals of some important papers of Fabrikant reprinted from original publications. Here we reprint this translation (without any changes at the request of Fabrikant's relatives) of the section "On experimental evidence for the existence of negative absorption" (where $N_{k}$ and $N_{j}$ are populations, $g_{k}$ and $g_{j}$ are the statistical weights of the levels $k$ and $j$, and $A_{r}$ is effective emission probability which were introduced in previous sections of [11]):

In this section, according to the theoretical considerations of previous sections, experiments that prove the existence of negative absorption (or equivalently, negative dispersion) are briefly discussed.

If the concentration of excited atoms is independent of the illumination, then the amount of energy absorbed in an infinitely thin layer will be proportional to $1-\frac{N_{k}}{N_{j}} \frac{g_{j}}{g_{k}}$, and it is the second term in this expression which accounts for the negative absorption. The absorption drops because of the occurrence of more emission events caused by the negative absorption.

Analysis becomes much more complex if the concentration of excited atoms depends on illumination, however.

At the first sight it may seem that, in the absence of quenching, negative absorption is of no significance at all. Indeed, the existence of negative absorption is equivalent to an enhancement of the emission probability, but if there is no quenching the emission probability has no effect on the radiation intensity...

This last assertion is valid totally for the full radiative flux emitted in all directions. In the direction of the incident beam, however, an increase in intensity due to the directional nature of negative absorption events should be observed. Because of the negative absorption, an angular redistribution of the radiation will occur. The presence of quenching only will enhance the effect. It should be noted therefore that our previous analyses of this problem underestimated the role of quenching. 
It is readily shown that in the presence of quenching the term responsible for negative absorption takes the form

$$
\frac{(1-a) A_{r}+B}{A_{r}^{\prime}+B} \frac{N_{k}}{N_{j}} \frac{g_{j}}{g_{k}}
$$

where $A_{r}^{\prime}$ is the emission probability increased due to the negative absorption, $B$ is the quenching probability, and $a$ is the solid angle subtended (seen S. L.) by the detector of radiation.

The above relation shows that for the experimental observation of negative absorption, it is necessary to first create the conditions that $a$ is small (a condition at which the influence of the discharge fluorescence will be also reduced). A favorable condition for observing negative absorption is a large value of $B$, i.e., strong quenching.

To prove the existence of negative absorption directly it would be desirable for the above term responsible for negative absorption to have a value in excess of unity, which requires that $\frac{N_{k}}{N_{j}}$ exceed the statistical weight ratio $\frac{g_{j}}{g_{k}}$.

Even though such concentration ratios are in principle achievable this requirement has never been fulfilled in discharges. Needless to say, this does not mean we must increase the current because the maximum concentration ratio will then be of Boltzmann type, i.e., the required inequality will not hold.

We believe that a realistic, but difficult, way to obtain the necessary conditions is, again the use of molecular impurities for selectively destroying lower lying levels. In this case, it is well known that fairly sharp resonances due to second-kind collisions are observed. Moreover, as shown above, radiation quenching, unavoidable in this case, will also be helpful. To evaluate the experimental conditions, the same Rosseland method may be utilized (in the original the author provides the reference to the previous chapter equation for the ratio of the exited state atom concentration to the concentration of atoms in the ground state $-\mathrm{S}$. L.).

In such experiments, we will obtain the intensity of output radiation exceeding that of the incident, so we may speak about direct experimental proof of the existence of negative absorption.

Later in our paper we will describe his experimental attempts to make a light amplifier as well as his 1951 Patent application.

To the idea of a population inversion Fabrikant came by analyzing the influence of different factors on the gas discharge emission intensity, and processes defining the distribution of atoms in excited states. But it seems that at the time of his thesis defense, a short section about "negative absorption" may have not been even reported in his oral presentation among other important results that Fabrikant discussed in it although the whole section (f) of the Chapter III of his written in 1939 and published in 1940 thesis [11] is devoted to negative absorption. The feeling of the author of the current paper (S. L.) is that, like Ladenburg (Section 3 of this paper), initially he may have considered his suggestions not so important to discuss them before, during and after defense with such gifted optical physicists as S. I. Vavilov [8]-[10] who supported him and L. I. Mandel'shtam [13] with whom he worked being a student. In the final Chapter V of his (published in 1940) thesis [11] entitled "The main results", "negative absorption" is not listed among them. So he may consider the section (f) of the Chapter III of his thesis with "negative absorption" not so important in comparison with other results of it [11]. In addition, in his later review on the optics of gas discharge [14], he, like Ladenburg in his review [15] on the same subject (see Section 3 of present paper), did not mention negative absorption at all.

The author of [7] who read available shorthand record of this defense, has different opinion suggesting that Defense Committee did not pay attention to Fabrikant's conclusions about negative absorption. In addition, she mentioned that Mandel'shtam and Papaleksi were not able to attend this defense [7].

\section{BRIEF HISTORY OF NEGATIVE ABSORPTION}

Before discussing the novelty of Fabrikant's ideas, we will briefly outline the state of the art at the time in understanding negative absorption. A. Einstein in his famous papers of 1916 [16]-[19] considering energy transfer from radiation to the molecule postulated his coefficients $B^{\prime}$ s connected with the physical phenomena of both absorption or induced (stimulated) emission processes. He called both processes with the same name "change the state under the irradiation" (in the original "Beide Vorgänge nennen wir 'Zustandsänderungen durch Einstrahlung' " ([16, 17], p. 51 of earlier 1916 paper and p.123 of its later 1917 reprint). In later 1923 paper ([20, 21], p. 302) A. Einstein and P. Ehrenfest called induced absorption "positive Einstrahlung" and induced radiation "negative Einstrahlung". See also [3, 22]. Einstrahlung in the sense of emission (negative absorption) was later called "induced emission" (for the first time by J. Van Vleck in a 1924 paper [23], p. 338). P. Dirac in his quantum theory of radiation of 1927 and $1930[24,25]$ proved the existence of induced emission postulated by Einstein. But negative dispersion, its connection to negative absorption, and the possibility of an intensity increase of the light passing through a medium with negative absorption was discussed already in 1924-1925 by H. Kramers [26, 27]. In his 1924 paper [26] introducing the "negative dispersion" terminology he wrote:

... one might introduce the following terminology: in the final state of the transition the atom acts as a "positive virtual oscillator" of relative strength $+f$; in the initial state it acts as a negative virtual oscillator of strength $-f$. However unfamiliar this "negative dispersion" might appear from the point of view of the classical theory, it may be noted that it exhibits a close analogy with the "negative absorption" which was introduced by Einstein, in order to 
account for the law of temperature radiation on the basis of the quantum theory.

Later, in the paper of 1925 [27], Kramers wrote:

... In the neighborhood of the frequency $v_{e m}$ of an emission line, the atom will then give rise to an anomalous dispersion of similar kind as in the case of an absorption line, with the difference that sign of the oscillating dipole induced in the atom is reversed. This so called "negative dispersion" is closely connected with the prediction made by Einstein, that the atom for such a frequency will exhibit a "negative absorption", i.e., light waves of this frequency, passing through a great number of atoms in the state under consideration, will increase in intensity.

Negative absorption (induced emission) was also discussed at this time by J. Van Vleck [23], R. Tolman [29, 30], and E. Milne [31] who derived in 1924 the formula of the "absorption integral" taking negative absorption into account. Milne found that the "absorption integral" is proportional to $1-\left(N_{2} / N_{1}\right)$, where $N_{2}$ and $N_{1}$ are number per $\mathrm{cm}^{3}$ of atoms in excited state and normal atoms. See also [4] (pp. 20-21) for more details. C. Füchtbauer who derived similar relation in 1920 neglecting negative absorption [32], measured such integral over the $\mathrm{Hg}$ resonance line in the presence of extraneous gas and found its decrease [33]. An explanation of this effect in terms of an elevated population in the higher state was given by A. Mitchell and M. Zemansky [34] (see [4], pp. 7-31, describing these papers). In [29] Tolman wrote (1924): “... molecules in the upper quantum state may return to the lower quantum state in such a way as to reinforce the primary beam by 'negative absorption' ". It should be noted that even one of famous Raman's papers of 1928 was called "The negative absorption of radiation" [35]. From today's knowledge we know that for the first time population inversion in the laboratory was achieved and induced emission was observed by E. Purcell and R. Pound during a short time interval in spin system using LiF crystal in 1950 (publication [36] of 1951) without amplification effect.

In 1928-1930, P. Ladenburg in Germany and his co-workers proved experimentally the existence of negative dispersion terms related to negative absorption of Kramers-Heisenberg's dispersion formula [37]. These results of Ladenburg were discussed in books [3, 4] and in papers [7, 38]. In 1928 Ladenburg transformed Kramers-Heisenberg's formula for dispersion [37] making it convenient for experimental verification [39]. He obtained a dispersion relation for refractive index $n$ :

$$
n-1=\frac{e^{2}}{4 \pi m c^{2}} \frac{\lambda_{k j}^{3}}{\lambda-\lambda_{k j}} N_{j} f_{k j}\left(1-\frac{N_{k}}{N_{j}} \frac{g_{j}}{g_{k}}\right),
$$

where $k$ and $j$ refer to any two stationary states ( $k$ being the higher one) with statistical weights $g_{k}$ and $g_{j}$ respectively, $N_{k}$ and $N_{j}$ are the numbers of atoms in the two states, $\lambda_{k j}$ is the wavelength of radiation emitted in the $k \rightarrow j$ transition and $f_{k j}$ is oscillator strength.
The term $\frac{N_{k}}{N_{j}} \frac{g_{j}}{g_{k}}$ is responsible for negative dispersion. In another paper of 1928 [40] H. Kopfermann and P. Ladenburg studying experimentally anomalous dispersion in a neon gas discharge found that this negative dispersion term $\frac{N_{k}}{N_{j}} \frac{g_{j}}{g_{k}}$ became more and more important with the current increase (See also their papers [41]-[44], P. Ladenburg and S. Levy's paper [45], and P. Ladenburg review [15]). They used very sensitive D. Rozhdestvensky hooks' method (hakenmethode) of studying anomalous dispersion of atomic vapors (See [46] cited in Kopfermann and Ladenburg's paper [42] (In 1928 Ladenburg visited Rozhdestvensky who was a Director of the Optical State Institute (GOI) in Leningrad at this time, saw his apparatus with an interferometer and asked for the detailed schematics of its construction [3]).

Regarding negative absorption, in [40], p. 376 Kopfermann and Ladenburg wrote that because of very strong connection between absorption and dispersion, it should be expected that with the process of negative absorption the anomalous dispersion sign would be opposite to the ordinary anomalous dispersion. They also draw a conclusion in a brief footnote at the end of this paper [40], p. 385 that for observation of negative dispersion the following inequality should be satisfied $N_{k} g_{j}>N_{j} g_{k}$ "that needs a special selective excitation". (The full text in German of this footnote is "Dazu müsste allerdings $N_{k} g_{j}>N_{j} g_{k}$ sein, was eine spezielle selective Anregung voraussetzt" - S. L.). This suggestion was not repeated anymore by these authors including Ladenburg final review [15] in which he writes [15], p. 234] that "... we find here the influence of negative dispersion only at very high currents, and this influence is the stronger the smaller the energy difference of the two levels considered, i.e. the longer the wavelength at which the anomalous dispersion is investigated". The same text was also written in paper [40] after reference to the above cited footnote of this paper [40]. This footnote was discovered later in laser era in [3] and reported only in Russian literature [3, 7] (by S. L.'s knowledge), maybe because paper [40] was published in a journal unusual for Ladenburg "Zeitschrift für Phys. Chemie". Fabrikant did not know about its existence as well, although in his paper of 1940 [11] Fabrikant cites several papers of Ladenburg and his co-workers of 1928-1930 [39, 42]-[44] including Ladenburg review [15], discussing some results of them (not connected with negative absorption).

In [47] C. Townes mentioned V. Ginsburg's letter to him regarding Ginzburg's professor of 1936-1937 S. Levy who had been well aware of "negative absorption" effects back in the 1930s and had told Ginzburg "create an overpopulation at higher atomic levels and you will obtain an amplifier; the whole trouble is that it is difficult to create a substantial overpopulation of levels". Levy was a Ladenburg's co-worker on negative dispersion experiments, see, e.g., [45] and emigrated from fascist Germany to the USSR and after that to USA after being fired in 1937 as former German worker [48]. In the USSR he translated for publication in 1934 Ladenburg's review [15].

Contrasting Ladenburg and Fabrikant's 1939 results we agree with the suggestion of [3] that at this time Fabrikant was the first who saw the possibility of light amplification at negative 
absorption conditions as the method to experimentally observing negative absorption. It seems that at that time nobody else thought on real experiments to observe negative absorption. We would like also to add that Fabrikant in his 1940 journal publication [11] of his 1939 thesis first wrote about possible real experiments and use of a gas medium, in particular to use "molecular impurities in a gas discharge for selectively destroying lower lying levels". In addition, in his section on negative absorption of his 1939 thesis [11], Fabrikant wrote (unlike Ladenburg) that "the required inequality of population inversion will not hold if we simply increase the current." Using a different approach independently from Ladenburg, Fabrikant also formulated the population inversion conditions and discussed an experiment for direct observation of induced absorption.

It should also be mentioned W. Lamb and R. Retherford 1950 paper [49]. In Appendix I [49], pp. 569-571, the authors analyzed the absorption of radiowaves by excited hydrogen atoms in a Wood discharge, and on p. 570 they wrote:

...if... $2^{2} \mathrm{P}_{3 / 2}$ is more highly populated, there will be a net induced emission (negative absorption!)... one would expect that $2 p$ levels would be about five to ten times more populated than the 2 s levels. In that case, one would expect to find a negative absorption, and as estimated below, a large one...

On p. 571 they wrote:

...it is possible that some appreciable departure from equipartition (statistical weights-S. L.) may exist, and that an absorption or induced emission could be detected. It is therefore highly desirable that a search for such effects should be made, especially under discharge conditions which do not favor equipartition.

\section{FABRIKANT, VUDYNSKY, BUTAEVA'S 1951 PATENT APPLICATION}

World War II interrupted Fabrikant's research. But already in 1945 he restarted his work at the Laboratory of New Light Sources of the All-Soviet-Union Electro-Technical Institute (VEI). Fabrikant jointly with senior scientist F.A. Butaeva carried out experiments trying to obtain negative absorption. Available documents show their notes about the 1950 experiments [7].

On 26 June 1951, V. A. Fabrikant and his co-workers (his former Ph.D. students) M. M. Vudynsky and F. A. Butaeva submitted a Soviet patent application entitled "Method for amplification of electromagnetic radiation (ultraviolet, visible, infrared and radio wavebands)" [50]. The application was submitted from Moscow Power Institute (Technical University) where Fabrikant was professor. According to Fabrikant [12, 51] (pp. 92 and 195 respectively), Butaeva started experiments, "but only in 1951 Vudynsky pointed out important possibilities of practical applications of this effect". On 16 July 1951 they submitted an addendum to their application at the Soviet patent office. In September of 1951 this application was sent for clearance and only on 16 December 1958 the application was declassified and approved. The Agency on Patents and Trademarks of the Institute of Industrial Property of Russian Federation has in its Archive 87 pages of documents related to this patent application [50]. It was acquired by the author of [7]. Among these documents are the initial patentapplication text of 1951, including schematics of the light amplifier and the act of application's classification [50], but no other documents of 1951-1954 according to [7]. But the approval document of 16 December 1958 which is also available, states that previous decision of 23 June 1955 to reject the 1951 application was a mistake. In 1959 a description of the patent was published in the Bulletin of Inventions [52].

In the text of the initial application it was written [50] (at the request of Fabrikant's relatives we reprint English translation from [12] of part of this initial 1951 patent application):

A method for amplification of electromagnetic radiation is proposed which exploits the induced emission phenomenon analyzed theoretically by A. Einstein in 1917. In this method the energy of the amplified radiation is not converted into other forms of energy. The method is suitable for amplification of ultraviolet, visible, infrared and radio frequency waves.

To implement the method a medium with a negative absorption coefficient for the radiation is produced. The radiative flux through such a medium increases in intensity - hence the amplification effect. The gain is given by $\exp (|K| L)$, where $K$ denotes the absorption coefficient and $L$ is the thickness of the layer.

A medium with a negative absorption coefficient results when its particles (for example, atoms or molecules) have a nonequilibrium distribution over energy levels. The concentration of particles in the upper energy states must exceed (with account for statistical weights) that at the lower states. As an example, a gaseous medium filling a suitable vessel is suggested, in which the required nonequilibrium condition is achieved, e.g., by an auxiliary radiation which excites particles to appropriate energy states; or by passing an electric current with a simultaneous use of impurities that selectively depopulate the lower states; or finally by passing a current modulated by using the ion-electron recombination effect to populate the upper states.

What we claim is a method for the amplification of electromagnetic radiation (ultraviolet, visible, infrared or radio wavebands), distinguished by the fact that the amplified radiation is passed through a medium, which by the means of auxiliary radiation or by other means, generates excess concentration, in comparison with the equilibrium concentration of atoms, other particles or their systems, at upper energy levels corresponding to excited states. 
Fabrikant writes the inversion conditions $\frac{\alpha_{2} \tau_{2}}{\alpha_{1} \tau_{1}}>\frac{g_{2}}{g_{1}}$, taking into account that $\frac{N_{2}}{N_{1}}=\frac{\alpha_{2} \tau_{2}}{\alpha_{1} \tau_{1}}$, where $\alpha_{1}$ and $\alpha_{2}$ is the number of atom excitation acts to lower $\alpha_{1}$ and higher $\alpha_{2}$ levels, $\tau_{1}$ and $\tau_{2}$ are radiation lifetime of these levels respectively. Fabrikant writes that this is the main inequality for the realization of a medium with negative absorption, which can be satisfied only under thermodynamic nonequilibrium conditions. He mentioned also that for negative absorption it is advisable that $\tau_{2}>\tau_{1}$ and it is possible to artificially reduce $\tau_{1}$.

On pp. 4-5 of [50] (p. 3 of initial patent application) Fabrikant writes "Amplification coefficient increases abruptly with increasing layer thickness $L$, therefore it is profitable to use recurrent (repeated, "povtornoye" in Russian - S. L.) beam propagation through the same layer."

It should be noted that the material submitted for the initial application is easy to distinguish from later material because of the applied stamps with the dates. The initial application of 1951 contains also schematics of "light amplifier" as well as description of an example of a particular gas medium (cesium vapors with impurities (p. 5 of [50], p. 3 of initial patent application) for such amplifier.

The materials of patent application [50] provide evidence of Fabrikant's efforts on light amplification experiments, so does the information from seminars initiated by him with discussion of his group work in this direction [7]. Reference [7] provides citation from one of the documents connected with Fabrikant's patent application [50], p. 73]: “Up to the end of 1950, these experiments started to give positive results, which were discussed in special colloquiums of the laboratory. This discussion's result was the 1951 application by V. A. Fabrikant, M. M. Vudynsky and F. A. Butaeva". After filing the patent application, the experiments continued [7]. In their notes of May 1963 Fabrikant et al. state that their patent application was discussed on February 1951 at a scientific seminar of a Special Physics Division of the Moscow Power Institute. To attract the wider scientific community into discussion of this problem, Fabrikant initiated in November 1951 a meeting of the Division of Technical Sciences of the Soviet Academy of Sciences [7].

The author of current paper (S. L.) has no information if S. I. Vavilov knew about Fabrikant's ideas and experiments. After harsh for USSR World War II (1941-1945), Vavilov was elected to the high position of the President of the Soviet Academy of Sciences in 1945, and had a difficult time on thinking how to save Physics in the USSR from destruction during Stalin's time (see, e.g., [53]). Although according to Fabrikant's paper about Vavilov [54], he saw Vavilov at Vavilov's scientific seminar. In that occasion Vavilov was complaining about his heart's problem just one day before his death (January 1951). S. L. suggests that details of his work on "light amplifier" which Fabrikant would surely had reported to Vavilov, appeared after Vavilov's death: specifically both the patent application of June 1951 and the results of his experimental attempts [55] (described below).

Summarizing this section, Fabrikant was the first who started to find the ways for population inversion in connection with light amplification, in particular, in gas media, offered three ways for it, and started experiments (jointly with Butaeva) with the purpose to reach light amplification in gas media (both in discharge and using optical pumping). The results of their experimental attempts were submitted in December 1957 and published in the book in 1959 [55]. The papers on the proposal of "gas lasers" by Sanders [56] on optical maser and Javan [57] on possibility of "negative temperature" in gas discharge were submitted 2 and 3 June 1959 and published on 15 July 1959

\section{FABRIKANT AND BUTAEVA'S EXPERIMENTAL ATTEMPTS ON LIGHT AMPLIFICATION}

According to [55], Fabrikant and Butaeva carried out two series of experiments to attain light amplification in the optical region. The first one was optical pumping of cesium vapors by the strong helium line $3888.65 \AA$ (these results on optical pumping of gas medium were submitted even earlier than paper [58] of Schawlow and Townes) and the second one was a gas discharge in a mixture of mercury and hydrogen.

In experiments with optical pumping of cesium vapors by $3888.65 \AA$ light, they investigated three lines of the fluorescence spectrum: $6983 \AA, 6723 \AA$ and $6973 \AA$. They selected these transitions because of their suggestion that $\tau_{2}$ of the upper levels $8^{2} \mathrm{D}$ should be longer than $\tau_{1}$ of lower levels $7^{2} \mathrm{P}$. The authors suggested that in this case the inequality $\frac{\alpha_{2} \tau_{2}}{\alpha_{1} \tau_{1}}>\frac{g_{2}}{g_{1}}$ should be satisfied. In these experiments, cesium vapors filled a Wood-type vessel with length $300 \mathrm{~mm}$ and diameter $28 \mathrm{~mm}$. Helium and other inert gases were added under several $\mathrm{mm}$ mercury pressure. Three helium lamps were the excitation source. Using a spectrograph, measurements of relative intensities of the three above mentioned lines were carried out at different excitation intensities by changing the current of helium lamps. It was observed that with the current increase the relative intensities of these lines were changed to the correct side, but the authors suggested that a too big value of the effect was suspicious. That is why they selected the second, more direct method. (Later cesium vapor lasing was obtained in near-IR, see, e.g., a description of a modern version of cesium laser with optical pumping [59]).

In the second experiment, Butaeva and Fabrikant investigated an excited gaseous mixture of mercury vapor and hydrogen between the levels $7^{3} \mathrm{~S}_{1}$ and $6^{3} \mathrm{P}_{0,1,2}$ of mercury. The population of $6^{3} \mathrm{P}$ levels should be reduced below that of the $7^{3} \mathrm{~S}_{1}$ level by the well known quenching action of hydrogen. The populations were investigated by measuring the transmission coefficient of the gas mixture at the wavelengths $5461 \AA$, $4358 \AA$ and $4047 \AA$, corresponding to the transitions $7^{3} \mathrm{~S}_{1}-$ $6^{3} P_{2}, 7^{3} S_{1}-6^{3} P_{1}$ and $7^{3} S_{1}-6^{3} P_{0}$. The mixture was excited by a d.c. discharge. With a discharge tube of length $36 \mathrm{~cm}$, amplification of about $10 \%$ was reported in the $5461 \AA$ and $4358 \AA$ lines for a discharge current of $70 \mathrm{ma}$, mercury vapor pressure $\sim 10^{-3} \mathrm{~mm}$ and hydrogen pressure $\sim 0.3 \mathrm{~mm}$ of mercury pressure. Adding helium enhanced the effect obtaining amplification also at $4017 \AA$. At the end of this paper the au- 
thors wrote that their attempts to explain obtained values of transparency by some other effects which were not connected with negative absorption, were not successful. But in paper [60] of 1961 on negative absorption in gas discharge (theory), Fabrikant cited the already published experimental paper on lasing in gas of Javan et al. [61] and did not mention his own experiments of [55].

Early in 1961, Oxford University physicists (Clarendon Laboratory), obtained an English translation of paper [55] by the author of [62] (see [63]) and immediately adapted their apparatus to search for gain on mercury transitions at the same conditions. At first, they measured optical gains of predicted magnitude for each of triplet lines, but later they discovered that it was the artifact connected with the so-called "superlinearity" of a photomultiplier tube (PMT) [63] and reported negative results in paper [64]. That it was not trivial at this time to identify this artifact in measuring gain in gas discharges, is shown by the Bell Labs example on anomalous results on gain on the transitions in the argon-ion laser which could not be made to lase [63] and which have been avoided when the author of [63] indicated the problem with PMT.

It should be noted that mercury vapor lasing was obtained later using different method of excitation —optical pumping [65].

In 1964 the Soviet Committee on Patents (Inventions) and Discoveries in addition to the 1961 patent, awarded Fabrikant, Vudynsky and Butaeva by "Discovery diploma" $N 12^{1}$. In 1965, the Soviet Academy of Sciences awarded Fabrikant the S.I. Vavilov's gold medal "For outstanding works on optics of gas discharge in which for the first time the phenomena connected with negative absorption have been investigated, and have been proposed for light amplification". Both these awards pass through extensive peer review including the Council of Division of Physics and Astronomy of the Soviet Academy of Sciences.

\section{SOME MOMENTS OF FABRIKANT'S BIOGRAPHY}

Here are some details of Fabrikant's biography [7]. Valentin Alexandrovich Fabrikant was born in 1907. His father was a professor of the Agricultural Academy. In 1925, after special high school, V.A. Fabrikant was accepted at the Department of Physics and Mathematics of Moscow State University. When he was a third-year student, G.S. Landsberg and L.I. Mandel'shtam offered him to check experimentally the quantum theory of recently discovered (by them) combination scattering (known as Raman scattering). Mandel'shtam asked him to use these measurements as a new method of defining Planck constant $h$. But a foreign paper appeared earlier with correct $h$ measurements by the same method and results of Fabrikant were not published.

\footnotetext{
${ }^{1}$ After S. I. Vavilov's et al. report to the Soviet government regarding the law protection of scientific discoveries, such law was approved in 1947. The first Discovery diploma was issued in 1957. From this time up to 1991, 403 Soviet Discovery diplomas were issued in all areas of scientific activity. All applications pass through reviewers with highest reputation.
}

Mandel'shtam and Landsberg were ready to hire the talented student after his graduation, but in March 1930 his father was arrested. (After Stalin's death all arrests were considered against the law and his name was fully exonerated. More details about arrests in Stalin's time see, e.g., book [53]). To give a better understanding of the environment, you may bear in mind that at this time also a relative of Mandel'shtam was arrested. V. A. Fabrikant was in shock and planned to leave the University. S. I. Vavilov saved him at this time. Already in 1925, during his lecture course on general physics, S. I. Vavilov noticed Fabrikant and continued to follow the further development of his skills. He observed Fabrikant's disastrous state and learned from him what had happened. After that, Vavilov, for whom it was impossible to employ Fabrikant directly into the P. N. Lebedev Institute under those circumstances, arranged for him to get a place as laboratory technician in All-Soviet-Union Electro-Technical Institute (VEI). Fabrikant received a Moscow State University diploma in October 1930 and after that was hired by VEI as a physicist of LightTechnology Laboratory. At this time several famous scientists from Academy of Sciences collaborated with VEI including Vavilov, Landsberg and Mandel'shtam. Mandel'shtam was even a director of VEI's theoretical laboratory (in addition to P. N. Lebedev Institute). Vavilov suggested a subject for scientific work to Fabrikant (connected with Vavilov's luminescence law) and every time visiting VEI discussed it, so Fabrikant evolved into Vavilov's disciple. When Fabrikant gave Vavilov his manuscript describing the results of this work for review, Vavilov edited it but refused to be its co-author. Vavilov wrote in Fabrikant's reference letter on his activity in the Light-Technology Laboratory of VEI in this period that scientific research in optics was started from scratch and that he and his two co-workers succeeded in a short time, and already during the first year, to carry out works of high scientific and technical merit. But in 1949, at the time of Stalin's struggling with "cosmopolites" [53], he was fired from VEI where he had a laboratory and carried out his main experiments (Butaeva worked there). It may be seen very strange because in 1947 he had been awarded the government Medal (Orden Znak Pocheta) for his work at VEI.

On Vavilov's recommendation, Fabrikant was invited as a lecturer (to replace Vavilov) at the Moscow Power Institute. He also helped him to resolve a conflict between unexperienced in teaching Fabrikant and his students who did not understand his lectures according the Fabrikant's paper about Vavilov [54]. Later Fabrikant became a Physics Department Chair of this Technical University (1943-1977), and devoted 50 year of his academic activity to it. In 1962, he introduced in his department a new specialty "Applied Physical Optics" to prepare engineers for laser development and applications. More than 700 students trained in lasers already graduated from this department. He is the person of merit for this educational institution. After his death in 1992, Physics Department of Moscow Power Institute was named in his honor.

In addition to his work on gas discharge and light amplifier, he was awarded (together with Vavilov, Butaeva et al.) the State prize of 1951 for his work in creation and research of luminescence lamp sources. In 1968 he became a full-fledged member (Academician) of the Soviet Academy of Pedagogi- 


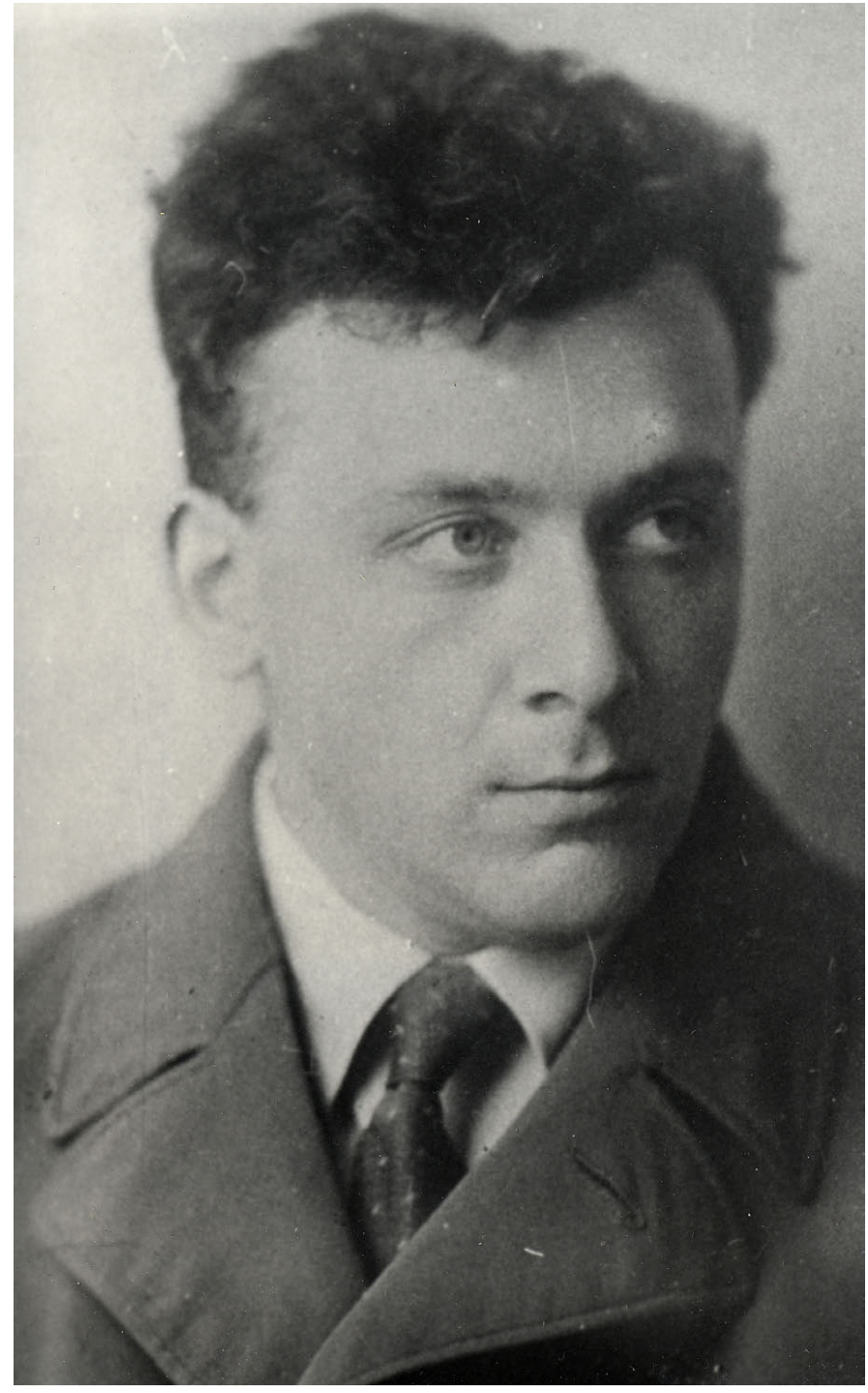

FIG. 2 Fabrikant's 1933 photograph at the time when he was a Laboratory Chair at All-Soviet-Union Electro-Technical Institute VEI.

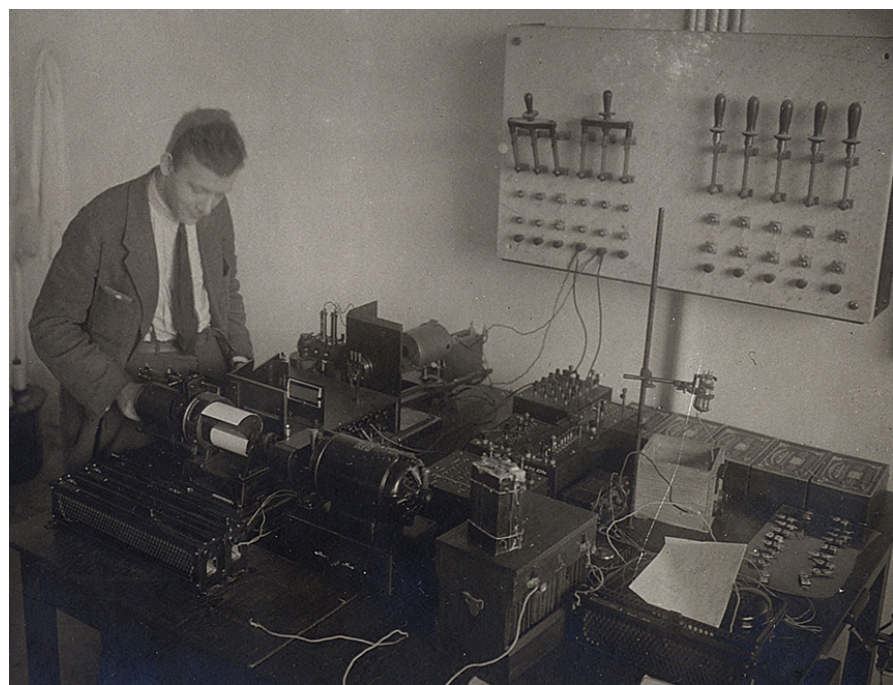

FIG. 3 Fabrikant's 1933 photograph at his laboratory of All-Soviet-Union ElectroTechnical Institute VEI.

cal Sciences. He published more than 250 papers. Figures 2-5 show photographs of V.A. Fabrikant at different time.

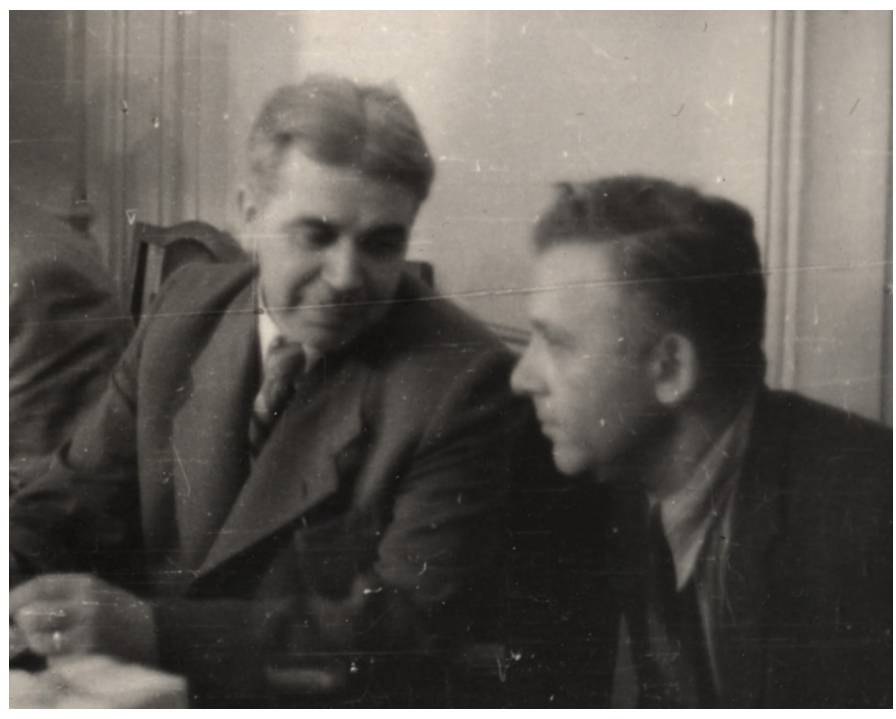

FIG. 4 Fabrikant (right) with S.I. Vavilov (left), 1946.

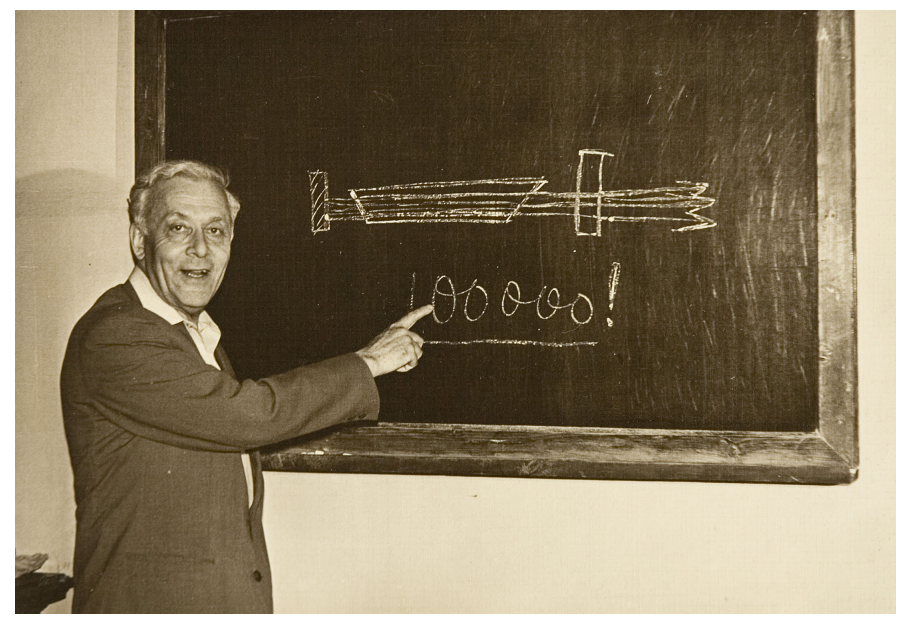

FIG. 5 Fabrikant delivering his lecture (1959-1960).

\section{CONCLUSION: FABRIKANT'S CONTRIBUTIONS TO PRELASER QUANTUM ELECTRONICS RESEARCH}

As a conclusion, we list here Fabrikant's contributions to prelaser era of quantum electronics:

1939 (from 1940 paper [11]):

- First publication with suggestion of experiments on light amplification directly proving the existence of negative absorption.

- First publication with suggestion of using molecular impurities and second-kind collisions to create population inversion in gas discharge.

- Formulation of population inversion conditions as a necessity for experimental observation of negative absorption (Kopfermann and Ladenburg formulated population inversion conditions in 1928 to prove the existence of negative dispersion term). 
1951 (from 1951 patent application [50] and paper [55]:

- Filing the patent application entitled "Method for amplification of electromagnetic radiation (ultraviolet, visible, infrared and radio wavebands)" based on started earlier experiments (at least from 1950) containing a schematics of "light amplifier", description of possible gas medium (cesium vapors with impurities), and population inversion conditions (thermodynamic nonequilibrium conditions).

- Suggesting three methods of obtaining population inversion in a gas medium: (1) by an auxiliary radiation (optical pumping) - independently from Kopfermann and Ladenburg; (2) in gas discharge with simultaneously use of impurities that selectively depopulate the lower states (first suggesting); (3) by modulated current using the ionelectron recombination effect to populate upper states (first suggesting).

- First suggesting to use repeated beam propagation through the same volume.

- First experimental attempts to obtain light amplification in gas media (both with optical pumping and with discharge). The results of these experiments in gas media were submitted earlier than papers of Sanders and Javan on proposal of "gas maser" and negative temperature in gas discharge $[56,57]$

\section{ACKNOWLEDGMENTS}

The author thanks A. E. Siegman for initiating this work, E. V. Gamsakhurdia-Fabrikant, K. G. Fabrikant, and G. R. Gamsakhurdia for providing the materials and photographs, for permission of publishing private materials and for help, E. I. Pogrebysskaya for providing some materials and discussions, A. W. Schmid, L. Clement-Rister, K. L. Vodopyanov, K. Tomilin, and D. A. Voloschenko for help, M. Bertolotti, N. V. Karlov and A. A. Manenkov for their comments, T. T. Basiev and S. B. Mirov for reading the manuscript.

\section{References}

[1] N. V. Karlov, 0. N. Krokhin, and S. G. Lukishova, "History of quantum electronics at the Moscow Lebedev and Ceneral Physics Institutes: Nikolaj Basov and Alexander Prokhorov" Appl. Opt. 49, F32-F46 (2010).

[2] S. Bagaev, 0. Krokhin, and A. Manenkov, "Pages from the history of quantum electronic research in the Soviet Union" J. Mod. Opt. 52, 1657-1669 (2005).

[3] I. M. Dunskaya, The Birth (Vozniknoveniye) of Quantum Electronics (Nauka, Moscow, 1974) in Russian.

[4] M. Bertolotti, Masers and Lasers. An Historical Approach (Adam Hilger, Bristol, 1983).

[5] C. H. Townes, How the Laser Happened. Adventure of a Scientist (Oxford University Press, Oxford, 1999).

[6] M. Bertolotti, The History of the Laser (Institute of Physics Publishing, Bristol and Philadelphia, 1999).
[7] E. I. Pogrebysskaya, "Valentin Alexandrovich Fabrikant: socialpolitical aspects of the biography of a physicist" in Scientific Society of Soviet Physicists. 1950-1960 years: Documents. Recollections. Investigations, V. P. Vizgin and A. B. Kessenikh, eds. 1, 484502 (Russian Academy of Sciences, S. I. Vavilov Institute for the History of Science and Technology, Russian Christian Academy, St-Petersburg, 2005) in Russian.

[8] B. M. Bolotowsky, Yu. N. Vavilov, "Sergei Vavilov, Honored in Russia, still little known in the West" Phys. Today 48:N1, 11,13 (1995).

[9] B. M. Bolotovskii, Y. N. Vavilov, and A. N. Kirkin, "Sergei Ivanovich Vavilov - the man and the scientist: a view from the threshold of the 21st century" Phys.-Usp+ 41, 487 (1998).

[10] B. M. Bolotovski, Y. N Vavilov, A. P Shmeleva, "Sergei Vavilov: luminary of Russian physics", CERN Courier, November 12, (2004). http://cerncourier.com/cws/article/cern/29202.

[11] V. A. Fabrikant, "The emission mechanism of a gas discharge" in Trudy (Proceedings) of VEI (the All-Soviet-Union Electro-Technical Institute), Electronic and Ion Devices 41, 236-296 (1940) in Russian.

[12] V. A. Fabrikant, Selected Works (MEI (Moscow Power Institute) Publishers, Moscow, 2007).

[13] V. V. Ragulsky "The most remarkable of the scientists: on the occasion of the 130th birthday of L. I. Mandel'stam" Phys.-Usp+ 52, 1177 (2009).

[14] V. A. Fabrikant, "Some questions of gas-discharge optics" Usp. Fiz. Nauk.+ 32, 1-25 (1947) in Russian.

[15] R. Ladenburg, "Dispersion in electrically excited gases" Rev. Mod. Phys. 5, 243-256 (1933).

[16] A. Einstein, "Strahlung-Emission und -Absorption nach der Quantentheorie" Deutsche Physik. Ges. Verhandl. 18, 318-323 (1916).

[17] The Collected Papers of Albert Einstein, A. J. Kox, M. J. Klein, and R. Schulmann, eds. (Princeton University Press, New Jersey, 1996)

[18] A. Einstein, "Zur Quantentheorie der Strahlung" Physik. Gesell. Zürich. Mitt. 18, 47-62 (1916).

[19] A. Einstein, "Zur Quantentheorie der Strahlung" Phys. Z. 18, 121128 (1917).

[20] A. Einstein, and P. Ehrenfest, "Zur Quantentheorie des Strahlungsleichgewichts" Z. Phys. Bd 19, 301-306 (1923).

[21] P. Ehrenfest, Collected Scientific Papers, M. J. Klein, ed., 485-490 (North-Holland, Amsterdam, 1959)

[22] H. P. Baltes, "On the validity of Kirchhoff's law of heat radiation for a body in a nonequilibrium environment" in Progress in Optics XIII, E. Wolf, ed., 1-25 (North-Holland, Amsterdam, 1976).

[23] J. H. Van Vleck, "The absorption of radiation by multiply periodic orbits, and its relation to the correspondence principle and the Rayleigh-Jeans law. Part I. Some extensions of the correspondence principle" Phys. Rev. 24, 330-346 (1924).

[24] P. A. M. Dirac, "The quantum theory of the emission and absorption of radiation" P. R. Soc. A 114, 243-265 (1927).

[25] P. A. M. Dirac, The Principles of Quantum Mechanics (Fourth Edition, Clarendon Press, 0xford, 1958).

[26] H. K. Kramers, "The law of dispersion and Bohr's theory of spectra" Nature 113, 673-674 (1924).

[27] H. K. Kramers, "On the behavior of atoms in an electromagnetic wave field" Skand. Mat. Kongress, 143-153 (1925).

[28] H. K. Kramers, Collected Scientific Papers 321-331 (North-Holland, Amsterdam, 1956). 
[29] R. C. Tolman, "Duration of molecules in upper quantum states" Phys. Rev. 23, 693-708 (1924).

[30] R. Tolman, Statistical Mechanics with Applications to Physics and Chemistry (The Chemical Catalog Co., New York, 1927).

[31] E. A. Milne, "The equilibrium of the calcium chromosphere" Mon. Not. R. Astron. Soc. 85, 111-141 (1924).

[32] C. Füchtbauer, "Die Absorption in Spektrallinien im Lichte der Quantentheorie" Phys. Z. 21, 322-324 (1920)

[33] C. Füchtbauer, G. Joos, and 0. Dinkelacker, “Über Intensität, Verbreiterung und Druckverschiebung vor Spectrallinen, insbesondere der Absorptionslinie 2537 des Quecksilbers" Ann. Phys.-Leipzig 71, 204-226 (1923).

[34] A. C. G. Mitchell, and M. W. Zemansky, Resonance Radiation and Excited Atoms 113-114 (Cambridge University Press, Cambridge, 1934).

[35] C. V. Raman, and K. S. Krishnan, "The negative absorption of radiation" Nature 122, 12-13 (1928).

[36] E. M. Purcell, and R. V. Pound, "A nuclear spin system at negative temperature" Phys. Rev. 81, 279-280 (1951).

[37] H. A. Kramers, and W. Heisenberg, "Über die Streuung von Strahlung durch Atome" Z. Phys. 31, 681-708 (1925).

[38] A. Kastler, "Masers and Lasers" Eur. J. Phys. 7, 69-76 (1986).

[39] R. Ladenburg, "Untersuchungen über die anomale Dispersion angeregter Gase. I. Teil. Zur Prüfung der quantentheoretischen Dispersionsformel" Z. Phys. 48, S15-S25 (1928).

[40] H. Kopfermann, and R. Ladenburg, "Experimenteller Nachweis der 'negativen Dispersion' " Z. Phys. Chem. A-Chem. T 139, S375-S385 (1928).

[41] H. Kopfermann, and R. Ladenburg, "Experimental proof of negative dispersion" Nature 122, 438-439 (1928).

[42] H. Kopfermann, and R. Ladenburg, "Untersuchungen über die anomale Dispersion angeregter Gase. II. Teil. Anomale Dispersion in angeregtem Neon" Z. Phys 48, S26-S50 (1928).

[43] H. Kopfermann, and R. Ladenburg, "Untersuchungen über die anomale Dispersion angeregter Gase. III. Teil. Übergangswahrscheinlichkeit und Dichte angeregte Atome im Neon; statistisches Gleichgewicht in der positiven Säule" Z. Phys. 48, S51-S61 (1928).

[44] H. Kopfermann, and R. Ladenburg, “Untersuchungen über die anomale Dispersion angeregter Gase. V. Teil. Negative Dispersion in angeregtem Neon" Z. Phys. 65, S167-S188 (1930).

[45] R. Ladenburg, and S. Levy, “Untersuchungen über die anomale Dispersion angeregter Gase. VI. Teil. Kontrollversuche für den Nachweis der negativen Dispersion: Absorption, anomale Dispersion, Intensitätsverteilung und Intensität verschiedener Neonlinien" Z. Phys. 65, 189-206 (1930).

[46] D. Roschdestwensky, "2. Anomale dispersion im Natriumdampf" Ann. Phys. 39, 307-345 (1912).
[47] C. H. Townes, "Early history of quantum electronics" J. Mod. Opt. 52, 1637-1645 (2005).

[48] V. L. Ginzburg, About Science, Myself and Others Ch. 19 (Institute of Physics Publishing, London, 2005).

[49] W. E. Lamb, Jr. and R. C. Retherford, "Fine structure of hydrogen" Phys. Rev. 79, 549-572 (1950).

[50] Materials of Soviet Patent application N576749, (1951-1964), 87 pages (Archive, Agency on Patents and Trademarks. Institute of Industrial Property of Russian Federation, Moscow).

[51] V. A. Fabrikant, "Albert Einstein and the light doctrine (to the centenary of birth)" Svetotechnika N3, 3 (1979) in Russian.

[52] V. Fabrikant, M. Vudynsky, and F. Butaeva, Bulletin of Inventions 20, (1959) in Russian.

[53] D Halloway, Stalin and the Bomb, The Soviet Union and Atomic Energy (1939-1956) (Yale University Press, New Haven at London, 1994).

[54] V. A. Fabrikant, "S.I. Vavilov as teacher of young scientists" Usp. Fiz. Nauk+ 117, 165-167 (1975) in Russian.

[55] F. A. Butaeva, and V. A. Fabrikant, "On the medium with negative absorption coefficient" in Investigations on Experimental and Theoretical Physics, in memory of Gregory Samuilovich Landsberg 6470 (Academy of Science, P. N. Lebedev Physical Institute, Moscow 1959) in Russian.

[56] J. H. Sanders, "Optical maser design" Phys. Rev. Lett. 3, 87-89 (1959).

[57] A. Javan, "Production of negative temperature in gas discharge" Phys. Rev. Lett. 3, 86-87 (1959).

[58] A. W. Schawlow, and C. H. Townes, "Infrared and optical masers" Phys. Rev. 112, 1940-1949 (1958).

[59] B. Zhdanov, and R. J. Knize, "Diode-pumped $10 \mathrm{~W}$ continuous wave cesium laser" Opt. Lett. 32, 2167-2169 (2007).

[60] V. A. Fabrikant, "Negative absorption coefficient produced by discharges in a gas mixture" Sov. Phys. JETP 14, 375-377 (1962); ZhETF 41, 524-527 (1961).

[61] A. Javan, W. R. Bennett, Jr., and D. R. Herriott, "Population inversion and continuous optical maser oscillation in a gas discharge containing a He-Ne mixture" Phys. Rev. Lett. 6, 106-110 (1961).

[62] B. A. Lengyel, "Evolution of masers and lasers" Am. J. Phys. 34, 903-913 (1966).

[63] C. Webb, "Laser research at Oxford in the 1960s" Opt. It Photon. News 14: $\mathrm{N}_{5}, 14-17$ (2003).

[64] J. H. Sanders, M. J. Taylor, and C. E. Webb, "Search for light amplification in a mixture of mercury vapour and hydrogen" Nature 193, 767 (1962).

[65] N. Djeu, and R. Burnham, "Optically pumped cw Hg laser at 546.1 nm" Appl. Phys. Lett. 25, 350-351 (1974). 